\title{
Estudio de la /d/ intervocálica en los corpus PRESEEA*
}

\author{
Irania Malaver ${ }^{1}$ \\ Universidad Central de Venezuela \\ José Antonio Samper Padilla² \\ Universidad de Las Palmas de Gran Canaria
}

\section{INTRODUCCIÓN}

Una de las características del PRESEEA ha sido la de buscar caminos que permitan unas comparaciones rigurosas desde un punto de vista sociolingüístico entre las distintas comunidades que se han integrado en el proyecto. Para ello se ha diseñado un tipo de muestra por afijación uniforme que permite unos cotejos directos: en cada comunidad habrá el mismo

* Esta publicación forma parte del proyecto de investigación "Estudio complementario de los patrones sociolingüísticos del español de España-Las Palmas de Gran Canaria" (Referencia: FFI2015-68171-C5-5-P), financiado por el Ministerio de Economía y Competitividad y por el Fondo Europeo de Desarrollo Regional (FEDER).

Para correspondencia, dirigirse a: Irania Malaver (irania.malaver@ucv.ve), Instituto de Filología "Andrés Bello" (IFAB), Facultad de Humanidades y Educación, Universidad Central de Venezuela, Edificio de la Biblioteca Central, Piso nº 11, Los Chaguaramos, 1080 Caracas, Venezuela.

2 Para correspondencia, dirigirse a : José Antonio Samper Padilla (joseantonio.samper@, ulpgc.es), Departamento de Filología Española, Clásica y Árabe, Edificio de Humanidades, Universidad de Las Palmas de Gran Canarias, Juan de Quesada, 30, 35001 Las Palmas de Gran Canaria, España. 
número de informantes según sexo, generación y nivel educativo, los tres condicionantes sociales que han de contemplar necesariamente todos los grupos de investigación. Es el tipo de muestra que se recoge en el siguiente cuadro:

Cuadro 1. Estratificación de la muestra-tipo por cuotas

\begin{tabular}{|l|c|c|c|c|c|c|}
\hline \multirow{2}{*}{ GI } & \multicolumn{2}{|c|}{$\begin{array}{c}\text { Generación 1 } \\
20-34\end{array}$} & \multicolumn{2}{c|}{$\begin{array}{c}\text { Generación 2 } \\
34-55\end{array}$} & \multicolumn{2}{c|}{$\begin{array}{c}\text { Generación 3 } \\
55 \text { años y más }\end{array}$} \\
\cline { 2 - 8 } & $\mathrm{H}$ & $\mathrm{M}$ & $\mathrm{H}$ & $\mathrm{M}$ & $\mathrm{H}$ & $\mathrm{M}$ \\
\hline Estudios primarios & 3 & 3 & 3 & 3 & 3 & 3 \\
\hline Estudios secundarios & 3 & 3 & 3 & 3 & 3 & 3 \\
\hline Estudios universitarios & 3 & 3 & 3 & 3 & 3 & 3 \\
\hline
\end{tabular}

Pero las comparaciones no requieren solo el mismo tipo de muestra en todas las ciudades; hay que asegurar también una delimitación homogénea de las variables dependientes y la consideración de unos factores explicativos comunes, pues eso va a permitir analizar con profundidad no solo qué alcance tiene un determinado fenómeno variable en las diferentes comunidades (que podrán resultar más o menos avanzadas) sino también cómo inciden los distintos condicionantes en la variación analizada, con la posibilidad de llegar a establecer unos caminos coincidentes y otros divergentes. Eso es algo que podremos constatar con los datos de la variable sobre la que tratamos en estas páginas.

Teniendo en cuenta lo dicho anteriormente, es natural que PRESEEA haya adoptado un sistema de codificación de las variables que se van a estudiar como paso previo a la puesta en marcha de su investigación. Esto quiere decir que el trabajo de los grupos se orienta en un primer momento a la discusión y puesta en común de la delimitación precisa de la variable dependiente (con los contextos específicos que se van a considerar) y de los condicionantes que se van a tener en cuenta para explicar de forma satisfactoria la variación observable. Con esos acuerdos se consigue la homogeneidad metodológica que permitirá dibujar un mapa representativo del mundo hispánico en su rica variedad geográfica y social. Es lo que se ha hecho con variables fónicas (como - d- intervocálica o-s implosiva), gramaticales (presencia/ ausencia de sujeto pronominal; personalización de haber; queísmo/dequeísmo...) y pragmáticas (atenuación). Esas codificaciones pueden encontrarse en la página web del proyecto (http://preseea.linguas.net/Inicio.aspx).

En lo referente a la variable (-d-), PRESEEA ha adoptado un tipo de codificación que ya había resultado apropiado para el estudio del fenómeno 
en España, tal como habían constatado los equipos integrados en una red de investigación que se ha venido reuniendo con periodicidad anual, desde 2004, para la programación de sus trabajos conjuntos. Nos referimos a los grupos de las universidades de Alcalá (que estudia Madrid), Granada, Las Palmas, Málaga y Valencia ${ }^{3}$. Análisis realizados con esos criterios fueron expuestos en las reuniones internacionales celebradas en Comillas (Cantabria, España) en 2007 y 2010; especialmente en este último año ya se pudieron presentar datos muy completos por parte de los distintos equipos que participaban en la investigación paralela. Los buenos resultados de esas investigaciones españolas mostraron la validez y eficacia del tipo de codificación empleado ${ }^{4}$, que, por tanto, se adoptó como base para el estudio panhispánico del fenómeno . Por ahora, además de los trabajos españoles, en América solo se ha realizado la investigación en la ciudad de Caracas, si bien tenemos noticias de que ya han empezado los análisis correspondientes en otras comunidades de las integradas en el proyecto.

En esta investigación general se consideran todas las realizaciones de /d/ que figuran entre dos vocales en posición interior de palabra. Esto significa que en el análisis no se tendrán en cuenta los ejemplos de $d$-inicial de palabra que puedan quedar en posición intervocálica por la tradicionalmente llamada "fonética sintáctica" ("la dalia", "madera de pino"). Con la delimitación anterior también quedan excluidos de nuestra consideración actual los casos en que /d/ sigue a una semivocal (caudaloso) o antecede a una semiconsonante (medio); estos últimos son contextos en los que la elisión de la dental tiene muy poca relevancia numérica, según indican estudios previos.

Esta delimitación de la variable dependiente contrasta con el entorno que ha sido más estudiado en las investigaciones sociolingüísticas anteriores, que prefirieron ceñirse al contexto en que $/ \mathrm{d} /$ interna sigue a la vocal acentuada y precede a /a/ u /o/ (seguidas o no de consonante), en palabras paroxítonas: $-v ́ S d v(c)$. La razón fundamental que había llevado a esta delimitación es que ese es el entorno donde el proceso de debilitamiento está más avanzado $\mathrm{y}$, por consiguiente, es el que ofrece mayor interés para el análisis de la variación en las realizaciones del segmento y para comprobar el papel de los

\footnotetext{
En un primer momento también participó el equipo de la Universidad de Lérida, que analizó los datos de la variable en el nivel socioeducativo superior. Desgraciadamente, después no pudo continuar en el proyecto conjunto. En los últimos años se ha integrado el grupo de la Universidad de Sevilla (ya se recogen algunas de sus aportaciones en el volumen que Sociolingüística Andaluza dedicó al profesor Pedro Carbonero).

Se encargaron de esta codificación Juan Villena y José Antonio Samper.

Los coordinadores del estudio de esta variable en el marco general del proyecto son Irania Malaver y José Antonio Samper.
} 
distintos condicionantes en esa fase del proceso. Por ejemplo, los estudios de Cedergren (1973), D’Introno y Sosa (1979), López Morales (1983), Samper (1990) o Alba (1999) se ciñeron al entorno citado. La delimitación que se ha realizado en el proyecto significa que nuestros datos no pueden compararse directamente con los de esos estudios anteriores que han definido la variable de forma distinta; por eso se ha de ser cauto al cotejar nuestros datos cuantitativos con los de las investigaciones previas, porque, en general, los índices de elisión serán más reducidos en el entorno más amplio seleccionado por PRESEEA.

Las variables explicativas que se contemplan son las siguientes:

(a) Lingüísticas: (1) acento, (2) número de sílabas de la palabra, (3) estructura acentual de la palabra, (4) entorno vocálico previo, (5) entorno vocálico posterior, (6) entorno consonántico en la palabra, (7) entorno consonántico secuencial, (8) categoría léxica, (9) categoría gramatical (morfológica), (10) homofonía, (11) funcionalidad, (12) límite morfemático, (13) difusión léxica y (14) asimilación al turno de palabra anterior.

(b) Estilísticas: (15) formalidad (tenor y estatus), (16) formalidad (tenor y edad), (17) formalidad (tenor y grado de proximidad), (18) especialización (campo), (19) tipo de discurso (estructura del discurso atendiendo a parámetros formales y semánticos sin tener en cuenta el grado de espontaneidad/planificación), (20) planificación y grado de espontaneidad, y (21) fase de la interacción.

(c) Sociales: (22) sexo, (23) edad, (24) nivel de estudios, (25) modo de vida, (26) comunidad de habla y (27) lengua habitual (en el caso de comunidades bilingües).

\section{RESULTADOS}

En este trabajo solo aportaremos los datos del entorno general, el correspondiente a la definición de la variable dependiente comentada anteriormente. A lo largo de la investigación el grupo de equipos españoles ha visto la necesidad de distinguir otros contextos para dar una explicación más ajustada del proceso y una razón que explique mejor el fenómeno de debilitamiento de la dental: (a) el entorno restringido, el más estudiado hasta ahora en las investigaciones sociolingüísticas, es decir, aquel en que la dental 
aparece precedida de vocal tónica en palabras paroxítonas; (b) el entorno bimorfemático, que tiene en cuenta los casos de /d/ gramatical o derivativa, y (c) el entorno más propicio, el del participio en -ado. Ahora, como hemos dicho, nos limitaremos a comentar los resultados generales y la incidencia de las variables explicativas en la elisión de la dental en el contexto que hemos señalado, es decir, el más amplio de los cuatro.

\subsection{Datos Generales}

En el gráfico 1 se muestran los índices de elisión de las seis ciudades en las que se ha estudiado la variable (d) intervocálica en todo el espectro social, con diferencias numéricas importantes entre ellas. Las comunidades representativas del español septentrional (Valencia y Madrid) son las más conservadoras, con un índice de pérdidas del 14\%; dentro de España las ciudades andaluzas orientales (Málaga y Granada) muestran un debilitamiento mucho más avanzado (33,6\% y $36 \%$, respectivamente). Las Palmas queda, dentro de las comunidades españolas, en una situación intermedia, con datos mucho más cercanos a los de Valencia y Madrid que a los de las ciudades andaluzas. Estos resultados iniciales muestran que el fenómeno de la elisión dental es más frecuente en la región del sur peninsular, confirmando las apreciaciones de los estudiosos que han clasificado el fenómeno como un rasgo andaluz (cfr., entre otros, Salvador 1964). Es llamativo que Caracas presente un índice de elisión superior incluso al de las ciudades de Andalucía Oriental, un dato que no confirma la idea de que la pérdida de -d- es más frecuente en la España peninsular que en América (cf. Alba 2015). Es necesario, pues, estudiar este fenómeno en las otras capitales americanas integradas en el proyecto PRESEEA; únicamente así será posible conocer de manera fidedigna la distribución geográfica de un fenómeno tan extendido en todo el mundo hispánico.

Gráfico 1. Porcentaje de elisión (entorno general) en los corpus PRESEEA

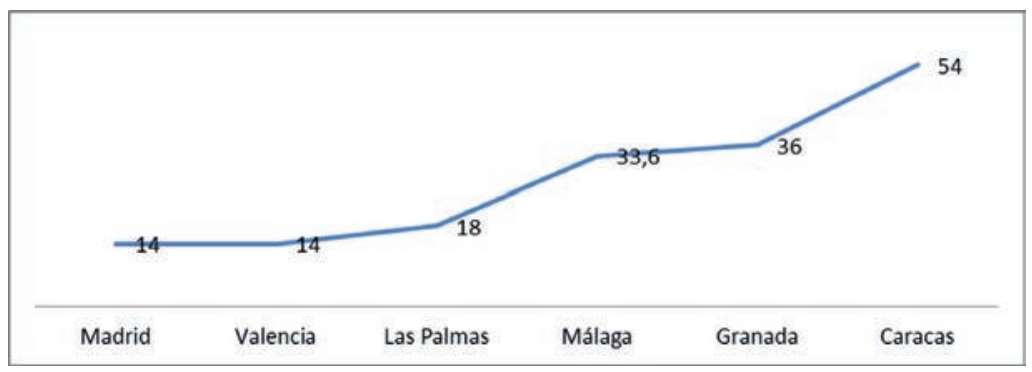


El cuadro 2 recoge los datos obtenidos en cada comunidad por las tres clases de variantes consideradas en la investigación: D-2, la plena [ $\delta]$, D-1, la variante debilitada $\left[^{\delta}\right]$ y D-0. la elidida $[\varnothing]$. En él puede verse, además de los resultados de la pérdida consonántica ya comentados, la distribución proporcional de las variantes que suponen mantenimiento de la dental: puede observarse que la realización más debilitada tiene importancia porcentual en la comunidad canaria y en la venezolana, pero su presencia es muy pobre en todas las ciudades peninsulares (en Madrid, donde se alcanza el promedio más alto, solo llega al 7,9\%). Esto implica que en la España peninsular, incluso en las comunidades andaluzas (las que tienden más a la elisión) se prefiere, cuando se pronuncia, una realización plena de la dental; por eso el índice de D-2 es más alto en Granada, por ejemplo, que en Las Palmas.

Cuadro 2. Resultados de las tres variantes de /d/ intervocálica en los corpus PRESEEA

\begin{tabular}{|l|c|c|c|}
\hline & Plena $[\delta]$ & Debilitada $\left[^{\circledR}\right]$ & Elidida $[\varnothing]$ \\
\hline Madrid & 77,7 & 7,9 & 14,2 \\
\hline Valencia & 83,4 & 2,5 & 14 \\
\hline Las Palmas & 58 & 24 & 18 \\
\hline Granada & 61,6 & 1,9 & 36,5 \\
\hline Málaga & 61,3 & 5,1 & 33,6 \\
\hline Caracas & 18 & 28 & 54 \\
\hline
\end{tabular}

\subsection{LOS FACTORES SOCIALES}

Comentaremos los resultados frecuenciales de los tres condicionantes sociales obligatorios (edad, nivel de estudios y sexo) en las seis comunidades estudiadas; aunque la codificación inicial prevé la inclusión de la variable modo de vida, no ha sido contemplada por todos los equipos y por eso prescindiremos de ella en esta presentación. 


\subsubsection{Factor edad}

Gráfico 2. Porcentaje de elisión según la edad

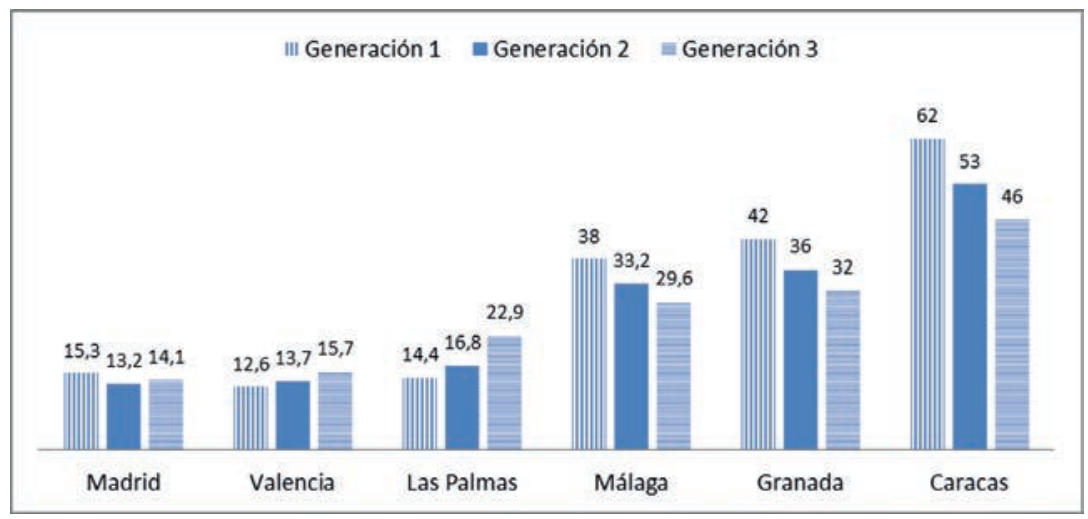

El gráfico 2 dibuja tres situaciones:

(a) Una ordenación lineal de las generaciones con un índice superior de elisión entre los jóvenes; nos encontramos ante comunidades en que el fenómeno sigue avanzando y en las que podría esperarse un aumento del proceso. Caracas, Granada y Málaga, precisamente las comunidades con un índice superior de elisión, son las tres ciudades que ofrecen este perfil. Las diferencias son más intensas en Caracas que en las dos capitales andaluzas.

(b) La ordenación lineal en sentido inverso al anterior; aquí el proceso se detiene en las generaciones más jóvenes, sobre todo en la primera. Ofrecen este ordenamiento las ciudades de Valencia y Las Palmas. Son comunidades de elisión moderada en las que, a partir de estos datos, no es previsible (sino todo lo contrario) un avance del proceso de debilitamiento extremo.

(c) Madrid ofrece una situación distinta: es la generación intermedia la que menos favorece la elisión $(13,2)$, frente a la más joven, que muestra el índice superior $(15,3)$. No obstante, las distancias no son acusadas. 


\subsubsection{Factor nivel de instrucción}

Gráfico 3. Porcentaje de elisión según el nivel de instrucción

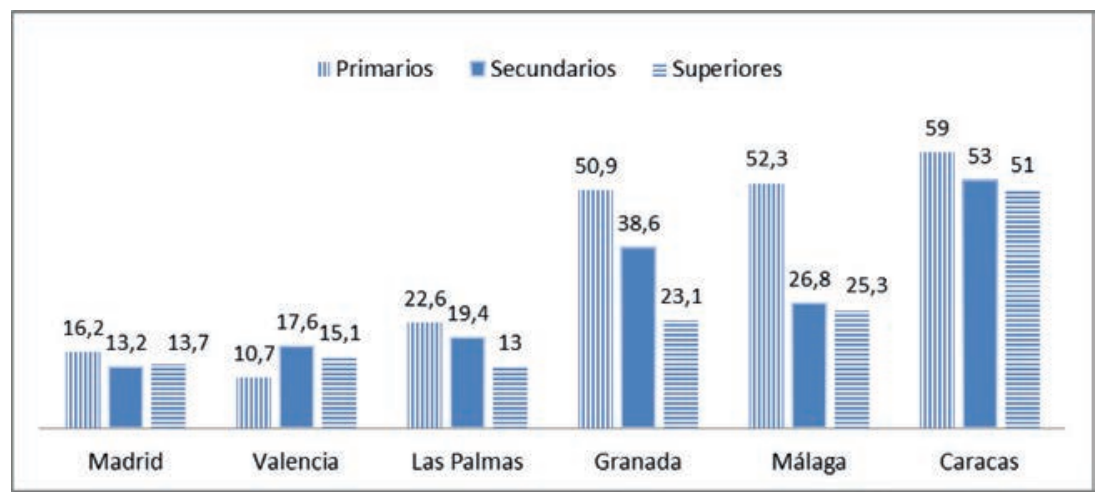

El patrón que vemos en el gráfico 4 refleja algo esperado de acuerdo con prácticamente todas las investigaciones previas (vid. Moreno Fernández 2004; Samper 2011: 114): la elisión es más intensa en los niveles más bajos (en las comunidades innovadoras los hablantes de estos niveles superan el $50 \%$ de D-0) y decrece en los otros dos. Pero las diferencias son más o menos marcadas: en las dos ciudades andaluzas vemos una importante distancia entre los niveles; en Caracas, por el contrario, los porcentajes de los tres estratos son relativamente cercanos.

En relación con el esquema anterior, de carácter panhispánico, solo cabe destacar la excepcionalidad que representa la comunidad valenciana, con un patrón curvilíneo que refleja el conservadurismo del nivel bajo en este proceso (con una diferencia destacable con respecto al nivel medio). 


\subsubsection{Factor sexo}

Gráfico 4. Porcentaje de elisión según el sexo

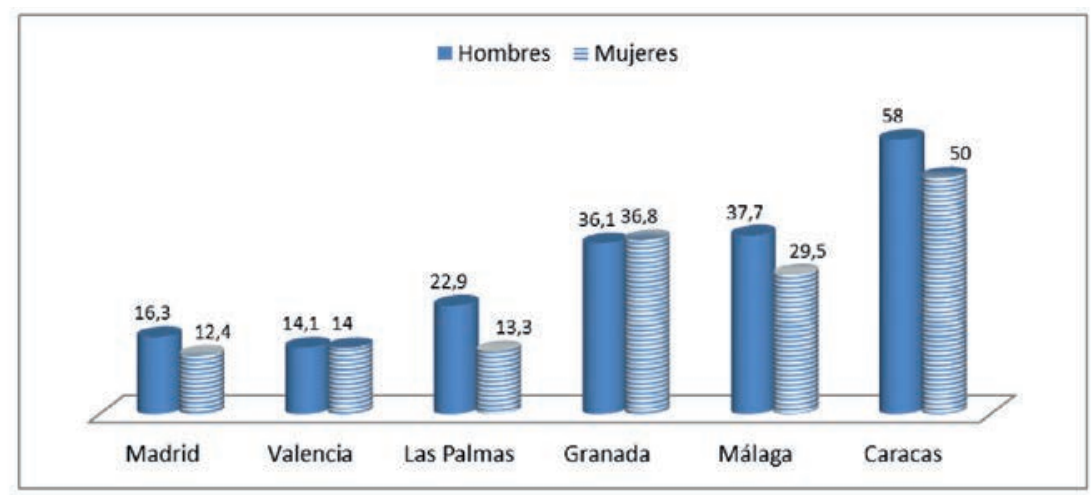

El gráfico anterior viene a mostrar que en prácticamente todas las comunidades la elisión de la dental se da más frecuentemente entre los hablantes masculinos. Este es un resultado que se refleja perfectamente en Las Palmas, Málaga, Caracas y Madrid, y que coincide con las conclusiones que ofrece Moreno Fernández (2004) al analizar los estudios realizados en 17 comunidades hispánicas. Granada y Valencia son, en este caso, las excepciones, si bien hay que reconocer que, aunque no reflejen el esperado mayor conservadurismo femenino en el proceso, tampoco muestran un índice superior de elisión entre las mujeres (los porcentajes son prácticamente iguales para ambos sexos).

\subsection{LOS FACTORES LINGÜÍSTICOS ${ }^{6}$}

Comentaremos en este apartado la incidencia de los factores lingüísticos que han sido seleccionados por el programa Goldvarb en determinadas comunidades $^{7}$. Las tres variables de carácter lingüístico que influyen de

\footnotetext{
6 Es mucho más limitada la incidencia de los factores estilísticos que se han contemplado en la investigación. Por ello prescindiremos en esta presentación de su estudio.

7 En este caso nuestros comentarios se limitarán a las ciudades de Caracas, Madrid, Valencia y Las Palmas. Los equipos de Granada y Málaga han trabajado fundamentalmente con el SPSS y no ofrecen los resultados probabilísticos de cada factor (sí dan, por supuesto, la significación estadística).
} 
manera más sobresaliente en la elisión de (d) intervocálica son la vocal antepuesta, la vocal pospuesta y la categoría gramatical de la palabra que contiene la dental. Son tres factores que han sido considerados en prácticamente todas las investigaciones sociolingüísticas previas sobre el segmento, y los datos de los distintos equipos vienen a confirmar los resultados anteriores, muchos de ellos de carácter panhispánico.

\subsubsection{Entorno vocálico previo}

Gráfico 5. Pesos probabilísticos de la variable entorno vocálico previo en tres corpus PRESEEA

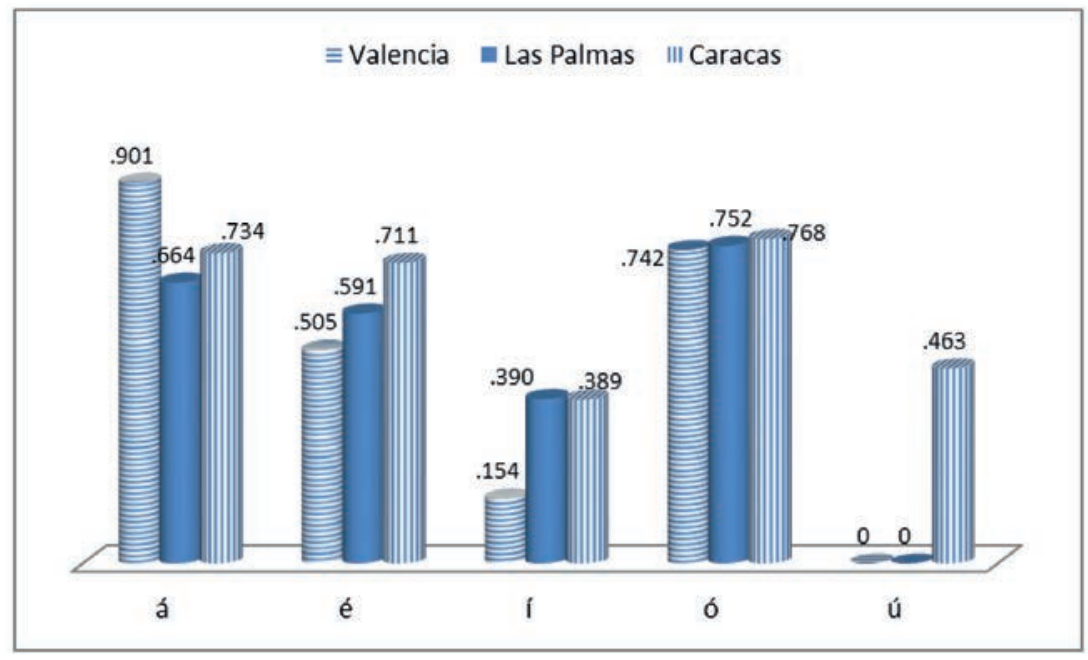

Las vocales precedentes que favorecen la elisión son las tónicas, aunque hay que descartar las vocales altas, pues uniformemente (ver los datos del gráfico anterior) bloquean la pérdida de la /-d-/, sobre todo la [ú] en las dos ciudades españolas (no tanto en la capital venezolana). Son [á] y [ó] las realizaciones vocálicas que ejercen un claro efecto en el proceso que analizamos: el de [ó] es más homogéneo (en torno a .750 en las tres ciudades); el de [á] es algo más diferenciado pues el índice que obtiene en Las Palmas y Caracas, aun siendo favorecedor, está notoriamente alejado del 0.901 que alcanza en Valencia una señal inequívoca de la mayor importancia que tiene allí el contexto -ado en el debilitamiento extremo de la dental. Los resultados que tenemos de Madrid también pueden considerarse parcialmente coincidentes: si se analizan los datos del contorno vocálico (Molina y Paredes 2014: 99), 
se observa que es la terminación -ado, la más frecuente en el corpus, la que alcanza una probabilidad superior, muy alta (0.92), en la elisión dental ${ }^{8}$.

\subsubsection{Entorno vocálico posterior}

En cuanto a la vocal pospuesta, los datos de Goldvarb que se exponen en el gráfico 6 muestran que en todas las comunidades, sin excepción, es la vocal [o] átona la que más incide en la elisión de /d/, algo que viene a confirmar el papel esencial de la terminación -ado cuando se estudia ese fenómeno. Realmente la mayor o menor frecuencia de elisión en esa terminación (y especialmente en el participio) puede ser un condicionante determinante en la diferenciación dialectal, pero en todas las zonas es un factor propiciador de la pérdida de la consonante.

Gráfico 6. Pesos probabilísticos de la variable entorno vocálico posterior en tres corpus PRESEEA

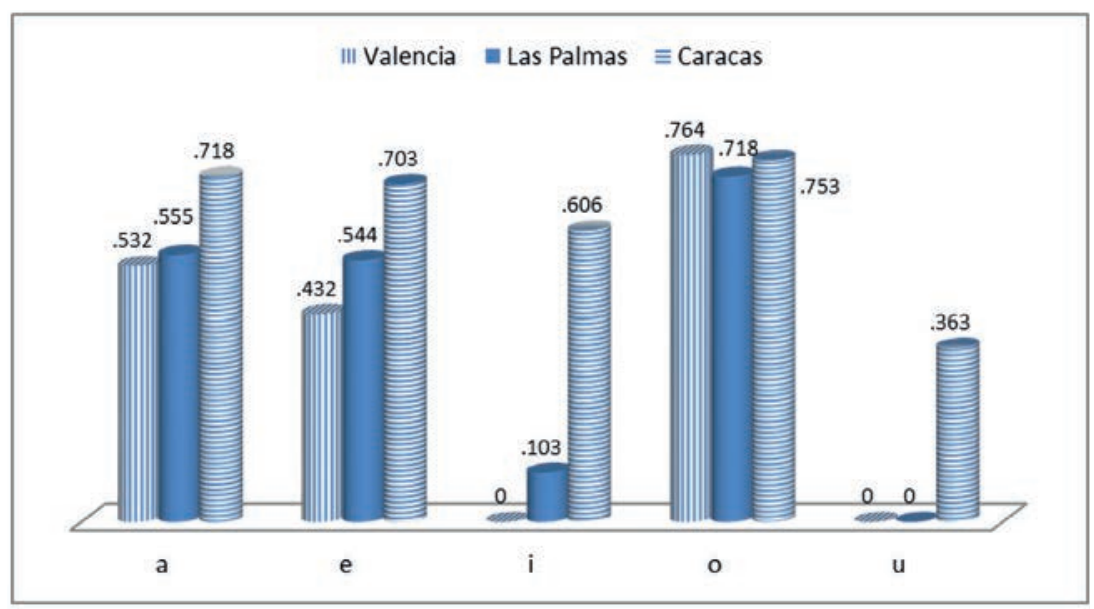

La vocal [a] átona pospuesta también favorece la elisión dental, pero con unos índices -excepto en Caracas- muy inferiores a los que alcanza [o]. Solo en la capital venezolana es también importante la significación de [e]

\footnotetext{
8 Es cierto que el índice de -ada no alcanza el $0.5 \mathrm{y}$, por tanto, no es un contexto que incida positivamente en la elisión, pero ha de tenerse en cuenta, en apoyo de nuestras consideraciones, la notoria diferencia de frecuencia de ambos entornos: de -ado se contabilizan 513 casos; de -ada, solo 38 .
} 
en el proceso de elisión, mientras que en las otras capitales cumple un papel mucho más limitado (favorable en Las Palmas y desfavorecedor en Valencia).

\subsubsection{Categoría gramatical (morfológica)}

Como esperábamos, la categoría de la palabra que contiene el segmento /d/ es una variable explicativa importante en todas las comunidades estudiadas. Para una formulación teórica que explique este proceso de debilitamiento es esencial tener en cuenta la incidencia de esta variable combinada con las dos que acabamos de comentar.

Es interesante constatar que, junto a resultados comunes que confirman los índices favorables obtenidos por algunos factores en investigaciones previas, hay algunas diferencias entre las comunidades que pueden estar indicando divergencias relevantes en el proceso de debilitamiento.

Gráfico 7a. Pesos probabilísticos de la variable categoría gramatical

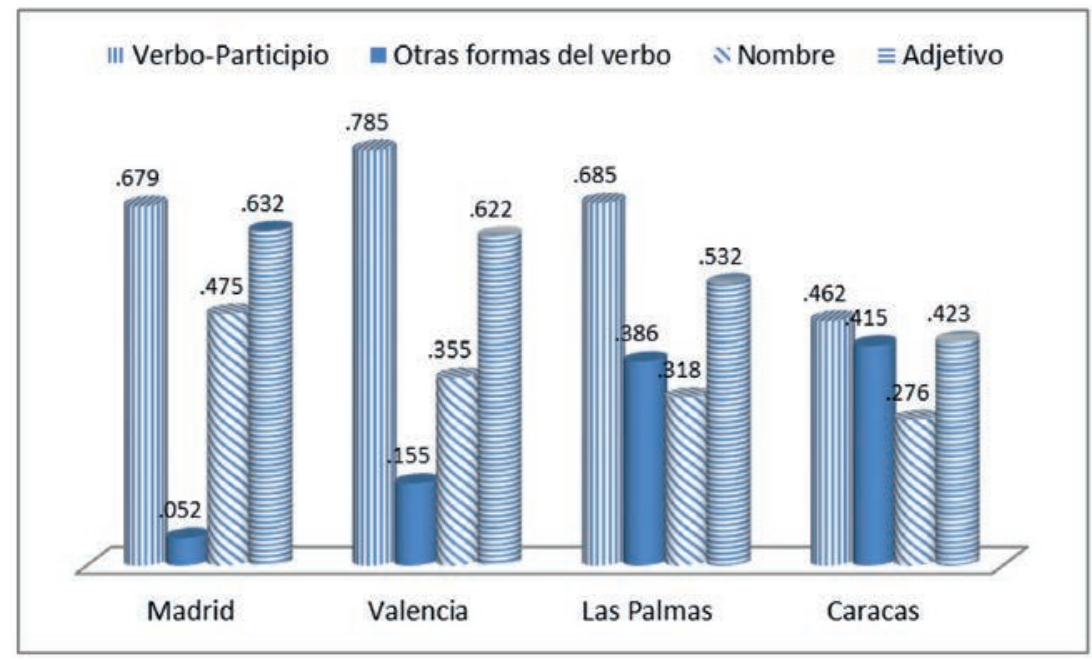


Gráfico 7b. Pesos probabilísticos de la variable categoría gramatical

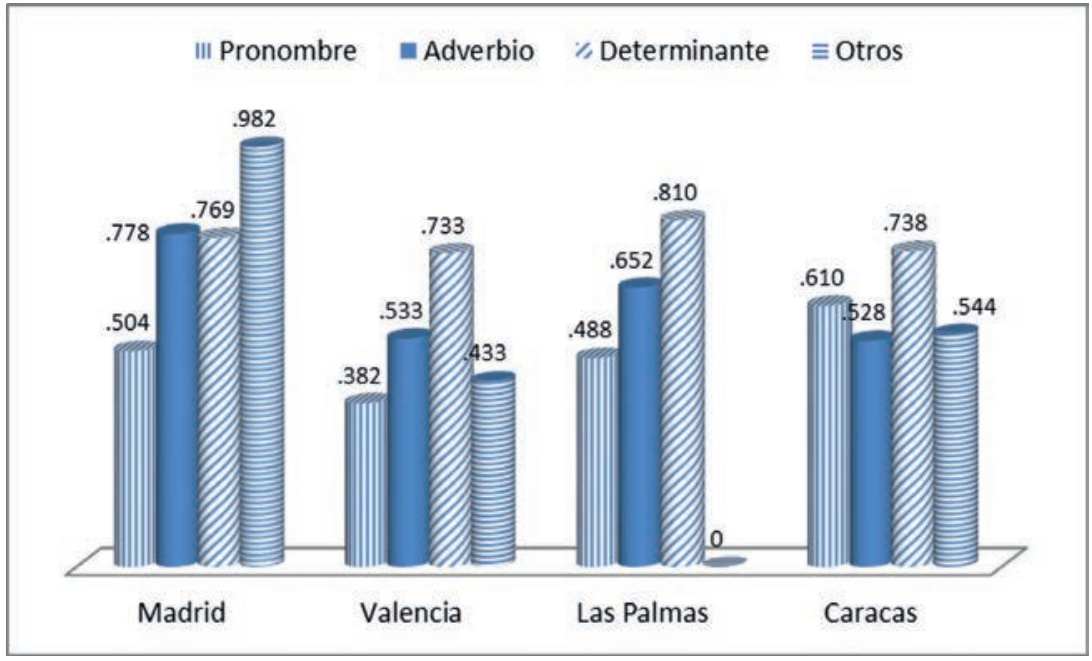

Entre los resultados comunes destaca la alta probabilidad alcanzada por la categoría determinante (gráfico $7 \mathrm{~b}$ ) en todas las comunidades; tal resultado está directamente relacionado con los altos índices de elisión de la -d- en el indefinido todo; en las cuatro ciudades la probabilidad de que no se pronuncie la dental se sitúa por encima de .700 . No parece tener un efecto similar el empleo de ese mismo elemento morfológico cuando funciona como pronombre, ya que esta categoría (los ejemplos no se reducen, por supuesto, a todo) no propicia la elisión ni en Valencia ni en Las Palmas, y en Madrid lo hace muy débilmente (.504). El adverbio es otra categoría en que los índices se sitúan por encima de .500, aunque con diferencias notorias en su incidencia en las distintas ciudades.

Otra característica común es que actúan como elementos desfavorecedores de la elisión en todas las comunidades (y por eso puede entenderse como una característica panhispánica) tanto el nombre como las formas verbales (sin contar en ellas las de participio), como muestra el gráfico $7 \mathrm{a}$.

No actúan de la misma manera, además de los pronombres citados anteriormente, ni el participio ni el adjetivo. La diferencia se establece nítidamente: las tres comunidades españolas seleccionan dichas categorías como claramente favorecedoras de la elisión (el índice más bajo es el de los adjetivos en Las Palmas), mientras que en Caracas tales categorías no alcanzan el .500 de probabilidad. La diferencia puede ser indicadora de 
que el proceso de debilitamiento no se ajusta a los mismos principios en la capital venezolana ${ }^{9}$ que en las ciudades españolas.

Un hecho particular llamativo es el altísimo índice que obtiene en Madrid la categoría Otros, algo relacionado, según los estudiosos de tal comunidad, con la elevada pérdida de la dental en la interjección joder.

\subsubsection{Otras variables lingüisticas}

Comentaremos superficialmente aquellas variables que han sido seleccionadas por Goldvarb al menos en dos o más ciudades e indicaremos qué factores son los que propician el grado extremo del debilitamiento dental:

1.3.4.1. En cuanto al acento, favorece la elisión en Madrid, Las Palmas y Caracas el que la vocal acentuada de la palabra sea la que precede inmediatamente a la dental, precisamente el contexto que coincide con el que ha sido estudiado más frecuentemente. En las tres ciudades actúa como un elemento claramente negativo para la pérdida el que /d/ forme parte de la sílaba tónica.

Gráfico 8. Pesos probabilísticos de la variable posición del acento

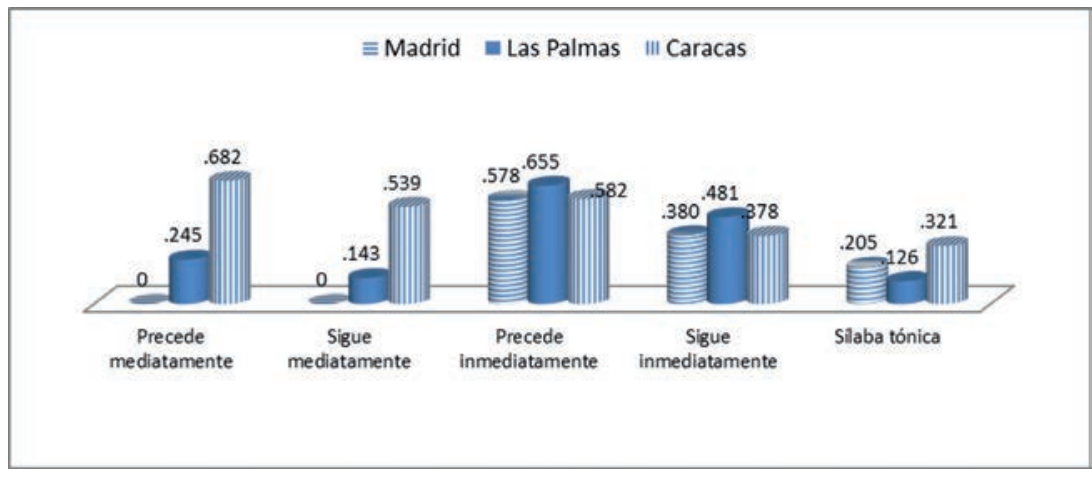

1.3.4.2. La palabra bisílaba es la que incide de forma positiva en la culminación del proceso en Madrid (.602), Las Palmas (.583) y Caracas (.610). La estructura trisílaba obtiene una probabilidad inferior a .500 en las tres comunidades, y la polisílaba solo resulta favorable en Madrid (.533).

\footnotetext{
9 Habría que comprobar si esa situación se produce también en otras ciudades
} hispanoamericanas. 
1.3.4.3. En cuanto a la estructura acentual, es la paroxítona la única favorecedora de la elisión, como puede verse en el gráfico 9. La aparición de la dental en una palabra proparoxítona u oxítona conlleva una alta probabilidad de mantenimiento; por eso el contexto analizado en casi todas las investigaciones sociolingüísticas previas fue el correspondiente a la estructura paroxítona.

Gráfico 9. Pesos probabilísticos de la variante estructura acentual (palabras paroxítonas)

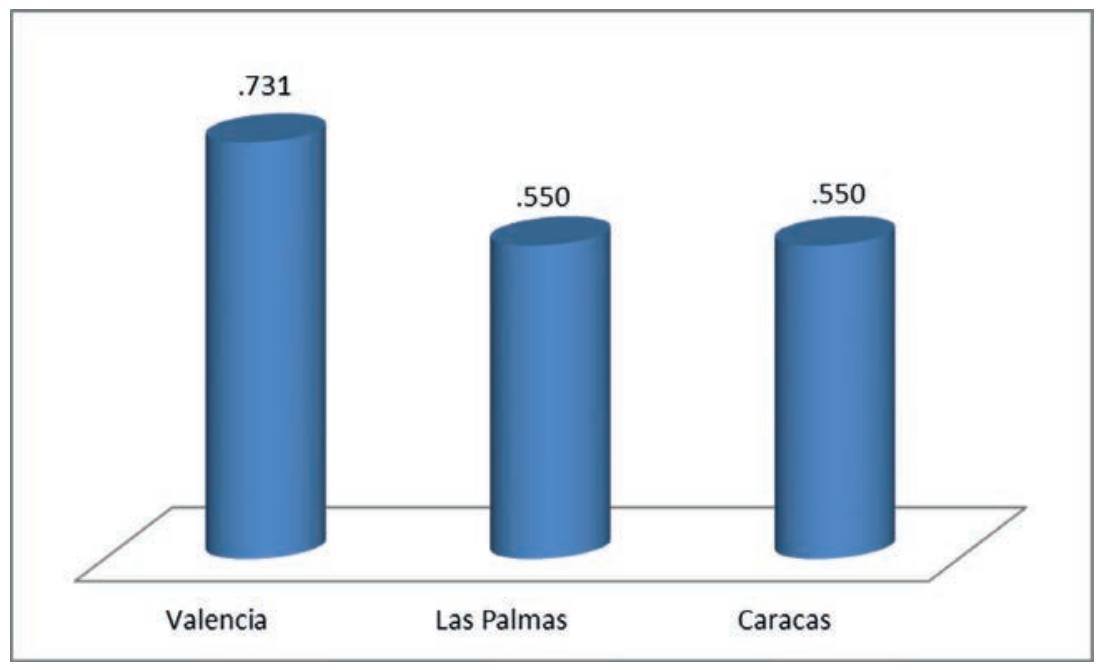

1.3.4.4. Solo en Caracas y Las Palmas resulta significativo el condicionante entorno consonántico en la palabra: en ambas comunidades favorece claramente la elisión el que en la palabra se haya dejado de pronunciar una /d/ anterior, mientras que el tipo de realización de la dental posterior no incide prácticamente en la posibilidad de que se mantenga o pierda la consonante analizada. 
Gráfico 10. Pesos probabilísticos de la variable entorno consonántico

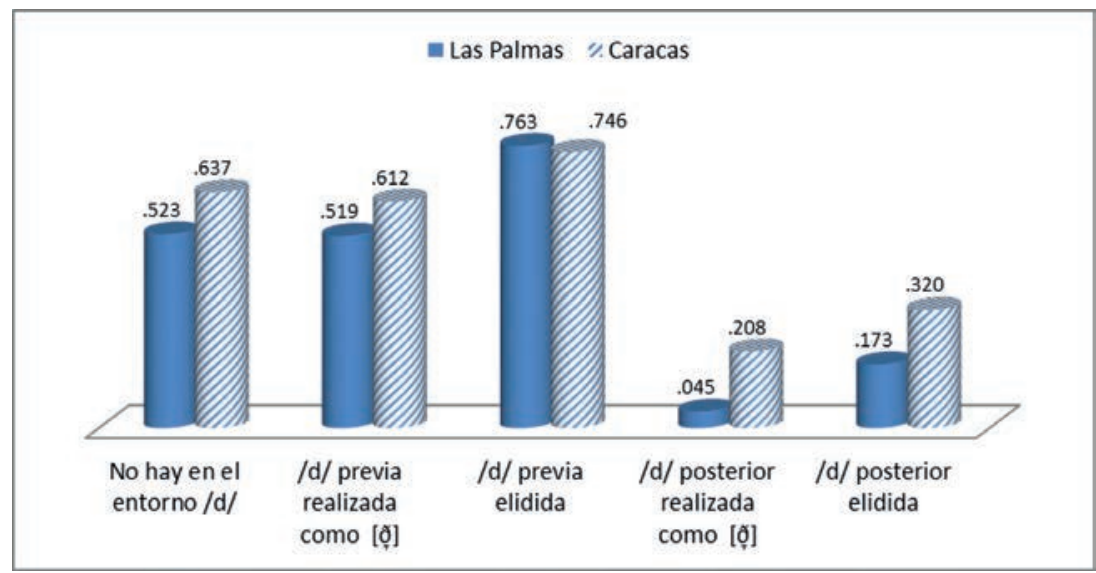

1.3.4.5. En tres comunidades resulta importante, desde el punto de vista de su significación estadística, la variable entorno consonántico en la secuencia. Tanto en Valencia como en Las Palmas y en Caracas favorece el proceso que analizamos el que haya otra /d/ elidida en el entorno secuencial.

Cuadro 3. Pesos probabilísticos de la variable entorno consonántico secuencial

\begin{tabular}{|l|c|c|c|}
\hline & Valencia & Las Palmas & Caracas \\
\hline No hay en el entorno /d/ & .414 & .481 & .480 \\
\hline $\begin{array}{l}\text { Hay en el entorno } \\
\text { consonántico secuencial /d/ } \\
\text { realizada(s) como [d] }\end{array}$ & .312 & .462 & .467 \\
\hline $\begin{array}{l}\text { Hay en el entorno consonántico } \\
\text { secuencial /d/ elididas }\end{array}$ & .708 & .722 & .553 \\
\hline
\end{tabular}

1.3.4.6. El cuadro 4 recoge los factores favorables para la elisión que coinciden en las ciudades de Valencia, Las Palmas y Caracas cuando se tiene en cuenta la variable homofonía. 
Cuadro 4. Pesos probabilísticos de la variable homofonía

\begin{tabular}{|l|c|c|c|}
\hline & Valencia & Las Palmas & Caracas \\
\hline $\begin{array}{l}\text { Homofonía entre nombre o } \\
\text { adjetivo y participio }\end{array}$ & .712 & .643 & .598 \\
\hline Otros casos de homofonía & .588 & .566 & .726 \\
\hline
\end{tabular}

1.3.4.7. Es muy relevante, porque puede estar marcando diferencias en el proceso entre las comunidades peninsulares, por un lado, y la venezolana y la canaria, por otro, el hecho de que Goldvarb no haya seleccionado el factor límite morfemático en estas dos últimas, pero que sí resulte uno de los condicionantes más destacados para la elisión en Valencia y Madrid.

Cuadro 5. Pesos probabilísticos de la variable límite morfemático

\begin{tabular}{|l|c|c|}
\hline & Madrid & Valencia \\
\hline Morfema & .700 & .738 \\
\hline Lexema & .363 & .273 \\
\hline
\end{tabular}

1.3.4.8. La variable difusión léxica ha resultado significativa en Madrid, Las Palmas y Caracas, pero no en Valencia. Si comparamos los índices que se obtienen en aquellas tres comunidades vemos que, además del mantenimiento de la dental en la forma vida (con índices muy negativos en todos los estudios), hay algunos otros resultados coincidentes: el más relevante, por la alta probabilidad de elisión de la dental que propicia, es el referido al adverbio todavía, dato que confirma la elevada frecuencia de la pronunciación toavía en grandes zonas del ámbito hispánico. También es positivo en las tres comunidades el índice alcanzado por el modificador todo, algo esperable después de conocer los datos que analizamos en cuanto a la categoría gramatical; asimismo no es sorprendente la menor incidencia de todo cuando funciona como núcleo: algo favorable en Las Palmas y Caracas, pero con un índice de .369 en Madrid. En cuanto a las otras formas, hay también coincidencia en el efecto favorable del verbo poder, con una alta frecuencia de elisiones en la tercera persona singular del presente de indicativo en muchas zonas del mundo hispánico; coinciden las dos ciudades españolas, frente a Caracas, en el efecto favorable de además (si bien en Madrid alcanza un índice mucho más alto que en la capital canaria), mientras que en la forma nada encontramos coincidencia en los índices madrileños y caraqueños, positivos, frente al de Las Palmas. Por último, sobresale la alta probabilidad de elisión que se da en Madrid en las formas del verbo 
quedar, algo que contrasta abiertamente con los índices, negativos, que se producen en Caracas y Las Palmas.

Gráfico 11. Pesos probabilísticos de la variable difusión léxica

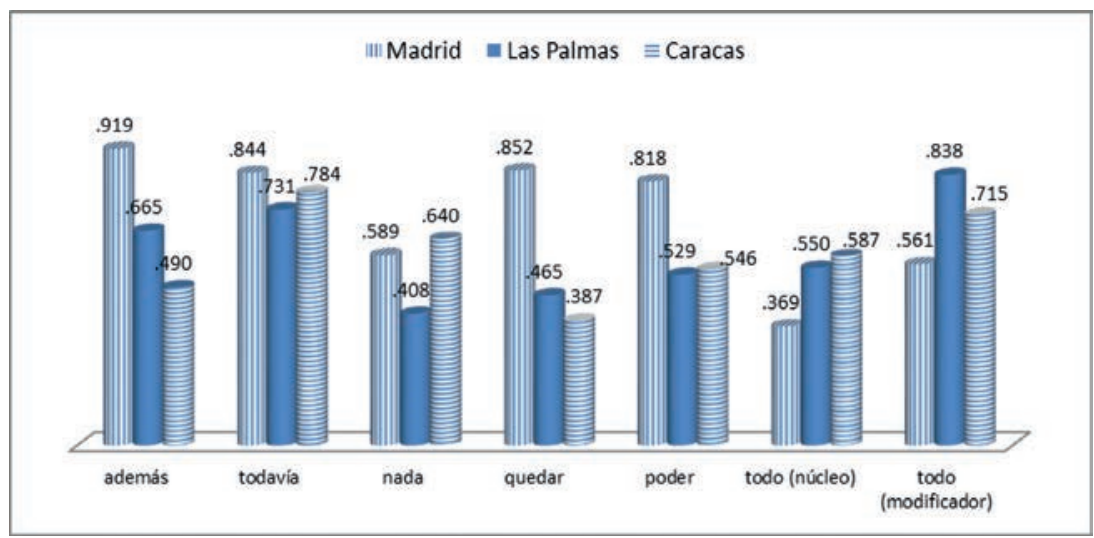

1.3.4.9. Valencia y Las Palmas coinciden en que también resulta significativa la variable asimilación al turno anterior (no sucede lo mismo en Caracas ni en Madrid). Favorece la elisión de la consonante el que en el turno previo haya una o más dentales elididas (con .576 de significación en Valencia y .747 en Las Palmas).

1.3.5. De la relación anterior han quedado excluidas por su falta de significación estadística en la mayoría de las comunidades que han trabajado con Goldvarb los factores lingüísticos categoría léxica y funcionalidad. Como vemos, son solo dos de las 14 variables propuestas las que no han resultado significativas al menos en dos o más ciudades.

\section{CONCLUSIONES}

Nos ha movido a redactar estas líneas el destacar la importancia que pueden alcanzar los trabajos conjuntos dentro del PRESEEA para conocer con fiabilidad y con datos actuales la situación de las variables sociolingüísticas más relevantes hoy en español. Hemos querido, con estos datos parciales de la 
variable /d/ intervocálica, mostrar nuestro convencimiento de que resultados iguales pueden lograrse con el trabajo coordinado con otras variables, algunas de las cuales ya están siendo objeto de estudio. Quisiéramos finalmente que estas líneas supongan un estímulo para que otros equipos se unan al propósito común y podamos aportar a nuestra comunidad una información que permita conocer mejor y más profundamente la variación que se constata en nuestra lengua.

\section{REFERENCIAS BIBLIOGRÁFICAS}

\section{Investigaciones PRESEEA \\ Caracas}

Malaver, Irania y Lorena Perdomo [en prensa]. La elisión de /d/ en posición intervocálica en la comunidad caraqueña. Boletín de Filología.

\section{Granada}

Moya Corral, Juan Antonio. 2009. Las tensiones del cambio lingüístico a propósito de la elisión de la /d/ intervocálica. En E. Montoro del Arco (ed.). El español del siglo XXI. Actas de las XIV Jornadas sobre la lengua española y su enseñanza, pp. 211-220. Granada: Universidad.

2012. Perdido. Resultados de la terminación -ido en el corpus PRESEEAGranada. Revista de Filología de la Universidad de La Laguna 30: 111-130.

Moya Corral, Juan Antonio y Emilio García Wiedemann. 2009. La elisión de /d/intervocálica en el español de Granada: factores lingüísticos. Pragmalingüística 17: 92-123.

Moya Corral, Juan Antonio, Emilio J. García Wiedemann, Esteban T. Montoro, Marcin Sosinsky, María Concepción Torres, Francisca Pose Furest y Elisabeth Melguizo. 2012. $\mathrm{La} / \mathrm{d} /$ intervocálica en Granada: factores lingüísticos y sociales. En Edyta Walluch y J. A. Moya (coords.). Español hablado. Estudios sobre el corpus PRESEEA-Granada, pp. 95-148. Varsovia: Instituto de Estudios Ibéricos e Iberoamericanos.

\section{Las Palmas}

Samper Padilla, José Antonio, Clara E. Hernández, Magnolia Troya, Ana Ma Pérez y MARTA SAMPER. 2010. Un estudio de variación fonética en el marco del PRESEEA: realizaciones de /d/ entre los hablantes de nivel universitario de Las Palmas de Gran Canaria, LEA 32: 5-34.

2011a. Elisión de /d/ en los niveles de estudios primario y universitario de Las Palmas de Gran Canaria. Comparación con otras comunidades de habla. En José Jesús de Bustos Tovar, Rafael Cano, E. Méndez y A. López (coords.). Sintaxis y análisis del discurso hablado en español. Homenaje a Antonio Narbona, vol I, pp. 409-424. Sevilla: Universidad de Sevilla.

2011b. Estudio sociolingüístico de la variación de -/d/- en la ciudad de Las Palmas de Gran Canaria" Comunicación presentada en el XVI Congreso Internacional de la ALFAL, Alcalá de Henares. 


\section{Madrid}

Molina Martos, Isabel y Florentino Paredes García. 2014. Sociolingüística de la elisión de la dental /d/ en Madrid (Distrito Salamanca). Cuadernos de Lingüística 2: 55-114.

2015. La conservación de la dental -/d/- en el distrito de Salamanca. En Ana María Cestero Mancera, Isabel Molina Martos, Florentino Paredes García (coords.). Patrones sociolingüísticos de Madrid, pp. 63-89. Berna: Peter Lang.

\section{Málaga}

Villena Ponsoda, Juan, 2012. Estatus, red e individuo. Fundamentos del análisis escalonado de la variación lingüística. Elisión de /d/ en el español de Málaga. En José F. Val Álvaro et al. (eds.), De la unidad del lenguaje a la diversidad de lenguas. Actas del X Congreso de Lingüística General, pp. 953-959. Zaragoza: Universidad de Zaragoza.

Villena Ponsoda, Juan Andrés, Francisco Díaz Montesinos, Antonio M. Ávila Muñoz y M. ${ }^{a}$ Cruz Lasarte Cervantes, 2011. Interacción de factores fonéticos y gramaticales en la variación fonológica: la elisión de /d/ intervocálica en la variedad de los hablantes universitarios en la ciudad de Málaga. En Yolanda Congosto y Elena Méndez (eds.). Variación lingüistica y contacto de lenguas en el mundo hispánico. In memoriam Manuel Alvar, pp. 311-359. Madrid/Francfurt: Iberoamericana/Vervuert.

\section{Sevilla}

JiMÉNEZ FERNÁNDEZ, RAFAEL. 2015. Sobre el mantenimiento y la pérdida de la /d/ intervocálica en el habla de Sevilla (sociolecto bajo). Sociolingüistica Andaluza 17: 193-209.

\section{Valencia}

Gómez Molina, José Ramón. 2012. Variación y cambios fónicos de la /d/ intervocálica en el español de Valencia: Proyecto PRESEEA-PRESEVAL. LEA 34: 167-196.

2013. Interacción de factores internos y externos en la pronunciación de la /d/ intervocálica. En José Ramón Gómez Molina (coord.). El español de Valencia. Estudio sociolingüístico, pp. 31-68. Berna: Peter Lang.

Gómez Molina, José Ramón y María Begoña Gómez Devis. 2010. Mantenimiento y elisión de la /d/ intervocálica en el español de Valencia. Verba 37: 89-122.

\section{ESTUDIOS COMPARATIVOS}

Gómez Molina, José Ramón, Isabel Molina Martos y Florentino Paredes García. 2012. La variación de la -/d/- intervocálica en comunidades de habla conservadoras de España: los casos de Madrid y Valencia. En Ana M. Cestero, Isabel Molina y Florentino Paredes (eds.). La lengua, lugar de encuentro. Actas del XVI Congreso Internacional de la Asociación de Lingüística y Filología de la América Latina, pp. 1975-1986. Alcalá de Henares: Universidad de Alcalá.

Moya Corral, Juan Antonio y Juan Andrés Villena Ponsoda (2011). Variables subyacentes y variables intervinientes en la variación de /d/ en el español de Andalucía. Comunicación presentada en el XVI Congreso Internacional de la ALFAL, Alcalá de Henares.

\section{REFERENCIAS BIBLIOGRÁFICAS}

Alba, Orlando. 1999. Elisión de la /d/ intervocálica postónica en el español dominicano. En Amparo Morales et al. (eds.). Estudios de lingüistica hispánica. Homenaje a María Vaquero. San Juan de Puerto Rico: Editorial de la Universidad de Puerto Rico, pp. 3-21. 2015. Madrid frente a Santo Domingo: la /d/ intervocálica y la /s/ implosiva. LEA 37: 5-32. 
Cedergren, Henrieta. 1973. La elisión de la /d/: un ensayo de comparación dialectal. Boletín de la Academia Puertorriqueña de la Lengua Española 7: 12-29.

D'Introno, Francesco y Juan Manuel Sosa. 1979. Elisión de la /d/ en el español de Caracas: Aspectos sociolingüísticos e implicaciones teóricas. Anuario de la Escuela de Letras $U C V:$ 33-61.

López Morales, Humberto. 1983. Estratificación social del español de San Juan de Puerto Rico. México: Universidad Nacional Autónoma de México.

Moreno Fernández, Francisco. 2004. Cambios vivos en el plano fónico del español: variación dialectal y sociolingüística. En Rafael Cano (coord.). Historia de la lengua española, pp. 973-1009. Barcelona: Ariel.

SALVADOR, GREGORIO. 1964. La fonética andaluza y su propagación social y geográfica. Presente y futuro de la lengua española, II, pp. 183-188. Madrid: Instituto de Cultura Hispánica.

Samper Padilla, José Antonio. 1990. Estudio sociolingüístico del español de Las Palmas de Gran Canaria. Las Palmas de Gran Canaria: La Caja de Canarias.

2011. Sociophonological variation and change in Spain. En M. Díaz-Campos (ed.). The Handbook of Spanish Sociolinguistics, pp. 98-120. Oxford: Blackwell Publishing Ltd. 